\title{
EchoGéo
}

55 | 2021

Espace et parentalité

\section{Géographies des parents, géographies des enfants}

Entretien avec Sonia Lehman-Frisch par Clément Rivière

\section{Sonia Lehman-Frisch et Clément Rivière}

\section{OpenEdition}

Journals

Édition électronique

URL : https://journals.openedition.org/echogeo/21089

DOI : $10.4000 /$ echogeo.21089

ISSN : 1963-1197

Éditeur

Pôle de recherche pour l'organisation et la diffusion de l'information géographique (CNRS UMR 8586)

Référence électronique

Sonia Lehman-Frisch et Clément Rivière, "Géographies des parents, géographies des enfants », EchoGéo [En ligne], 55 | 2021, mis en ligne le 30 mars 2021, consulté le 11 août 2021. URL : http:// journals.openedition.org/echogeo/21089; DOI : https://doi.org/10.4000/echogeo.21089

Ce document a été généré automatiquement le 11 août 2021.

EchoGéo est mis à disposition selon les termes de la licence Creative Commons Attribution - Pas d'Utilisation Commerciale - Pas de Modification 4.0 International (CC BY-NC-ND) 


\title{
Géographies des parents, géographies des enfants
}

\author{
Entretien avec Sonia Lehman-Frisch par Clément Rivière
}

\author{
Sonia Lehman-Frisch et Clément Rivière
}

Clément Rivière (CR). Sonia Lehman-Frisch, vous avez consacré plusieurs années de vos recherches aux expériences urbaines des enfants. Pourriez-vous revenir sur les conditions d'émergence de la recherche sur (et avec) les enfants en géographie, en France et ailleurs?

Sonia Lehman-Frisch (SLF). Il faut d'abord rappeler que la géographie, et les sciences sociales en général, ont commencé à s'intéresser aux enfants dans les années 1970 et 1980. En France comme aux Etats-Unis, plusieurs travaux pionniers ont émergé en géographie et en sociologie en particulier, et je voudrais brièvement rappeler les deux grandes directions qu'ils ont tracées et qui sont liées à leur contexte d'émergence. Un certain nombre d'entre eux s'inscrivent en effet dans le contexte du renouvellement épistémologique de la géographie et de l'émergence de la "géographie de la perception", qui s'inspire beaucoup de la psychologie environnementale. Pour eux, l'espace est une construction mentale qu'il s'agit de saisir, et les représentations de l'espace varient d'un individu ou d'un groupe d'individus à l'autre. Les enfants représentent un groupe social particulier, pour lequel il s'agit de saisir les processus cognitifs d'appréhension de l'espace en fonction de l'âge et des étapes du développement de l'enfant. Un autre ensemble de travaux s'est quant à lui davantage intéressé aux pratiques urbaines des enfants, interrogeant la place des enfants en ville et l'adaptation ou l'inadaptation de l'environnement urbain pour cette population particulière. C'est le cas de la sociologue MarieJosé Chombart de Lauwe en France, ou de Kevin Lynch aux Etats-Unis. Cette opposition est bien sûr un peu caricaturale car les deux approches sont liées en réalité.

Dans l'ensemble, ces travaux sont restés relativement marginaux et il a fallu attendre les années 1990 pour que se produise ce que j'appelle le «baby boom » des Children's Geographies, dans la sphère scientifique anglophone principalement - en France, ce n'est qu'à la fin des années 2000 que les sciences sociales se saisissent réellement de 
la question du rapport à l'espace des enfants. C'est l'apparition d'un nouveau contexte qui explique ce brusque essor. Premier élément de contexte, les sciences sociales, qui ont pleinement réalisé les enjeux scientifiques liés à l'urbanisation accélérée de la population mondiale prennent désormais conscience que près du tiers de cette population sont des enfants, soit un milliard de citadins, comme le révèle l'UNICEF à ce moment-là. Deuxième élément de contexte, la Convention internationale des droits de l'Enfant, adoptée en 1989, place les enfants sous les projecteurs de la scène internationale. Cet événement majeur contribue à légitimer l'intérêt scientifique pour une catégorie de population jusqu'ici négligée par la recherche. Troisième élément, cette double prise de conscience se traduit concrètement par le lancement de vastes programmes de recherche au Royaume-Uni et aux Etats-Unis, ainsi qu'à l'échelle internationale (un vaste programme est lancé par l'UNESCO par exemple).

Cette nouvelle demande sociale se produit dans un contexte de renouvellement scientifique et intellectuel. Les années 1990 sont en effet le temps d'une profonde réflexion épistémologique sur le concept d'enfant. Suite aux travaux de l'historien Philippe Ariès, les sciences sociales contestent la vision naturaliste de l'enfance, qui la présente comme une donnée biologique, et elles montrent au contraire que l'enfance est un construit social et historique : le concept d'enfant a changé dans le temps, il varie aussi dans l'espace selon les sociétés considérées, et il n'est pas le même non plus selon les groupes sociaux observés. Ce qui pose d'ailleurs la vaste question des limites de l'enfance... Autre remise en cause épistémologique, l'enfant n'est plus considéré comme un être en devenir que les forces de la socialisation transforment en être accompli, c'est-à-dire en adulte, mais les sciences sociales le pensent désormais comme un acteur social à part entière. Il a fallu ce renversement dans la manière de penser l'enfant, ou plutôt les enfants, pour que les sciences sociales puissent affirmer la légitimité de ce sujet de recherche et se lancer dans l'exploration de toutes ses potentialités scientifiques.

C'est donc la convergence de ces éléments de contexte sociaux, politiques et scientifiques qui explique l'émergence des Children's Studies à partir des années 1990, et même, à l'intérieur de ce champ foisonnant, d'un courant dynamique consacré spécifiquement aux Children's Geographies. Ce courant, qui possède ses propres colloques, ses ouvrages de référence (souvent issus de ces derniers), et même ses revues spécialisées (notamment la revue éponyme Children's Geographies, créée en 2003), s'intéresse spécifiquement à la dimension spatiale de l'expérience quotidienne des enfants : il étudie la manière dont l'espace structure les vies enfantines et dont, inversement, les enfants interprètent et produisent leurs territoires par leurs pratiques et leurs représentations.

CR. D'un point de vue méthodologique, des enjeux spécifiques sont-ils associés à cette approche géographique des enfants?

SLF. Oui, ces recherches se sont développées avec l'idée que les enfants ne sont pas une population d'enquêtés comme les autres. Il y a deux manières d'étudier le rapport à l'espace des enfants. On peut le faire à partir de la perspective des adultes : en menant des entretiens auprès des différents acteurs impliqués dans la vie des enfants (parents, enseignants ou autres acteurs), ou par l'observation directe dans différents lieux (parcs, école, logement, etc.), on peut obtenir des données sur les représentations et les pratiques des enfants. Ces informations, aussi précieuses 
soient-elles, sont indirectes, passées au filtre de l'interprétation des adultes (ce qui en soi constitue d'ailleurs un matériau intéressant, on y reviendra tout à l'heure). Une autre solution, et c'est plutôt cette approche qui a été privilégiée par les chercheurs s'inscrivant dans la perspective des Children's Geographies, consiste à faire s'exprimer les enfants eux-mêmes sur leur rapport à l'espace. Cette perspective, nouvelle, pose deux séries d'enjeux méthodologiques.

La première série d'enjeux est liée à la question de la capacité des enfants à exprimer leur propre rapport à l'espace. Si l'entretien individuel est l'un des principaux outils des sciences sociales, y compris de la géographie, pour saisir le rapport à l'espace des enquêtés, les chercheurs ont vite réalisé ses limites quand il s'agit d'interroger des enfants, en particulier les plus jeunes. Cela a eu deux conséquences. D'abord, cela contribue à expliquer que les recherches conduites auprès des jeunes enfants soient restées relativement rares pendant longtemps par rapport à celles portant sur les adolescents par exemple, en lien avec l'idée qu'ils n'auraient pas les compétences suffisantes pour exprimer verbalement leurs pratiques et leurs représentations. En même temps, et c'est l'autre conséquence, les chercheurs ont cherché à diversifier les méthodes, ou pour le dire autrement, à proposer aux enfants, y compris les plus jeunes, une grande variété d'outils pour leur permettre de montrer leur perspective sur l'espace. Parmi ces méthodes, les méthodes visuelles tiennent une place particulière: les dessins, les cartes mentales, les photos, et plus récemment les vidéos, ont été largement utilisés dans ces recherches. Il faut rappeler qu'elles ont été développées par la psychologie environnementale : cartes, dessins et photographies sont très tôt apparus comme des outils essentiels pour étudier les processus cognitifs d'appréhension de l'espace. Dès le début de la recherche collective à laquelle j'ai participé, nous avons été confrontés nous aussi à cette question. Nous avons fait le choix de mettre en place un dispositif mixte : des dessins d'une part, et d'autre part des entretiens appuyés sur un ensemble de photographies et sur le dessin produit par l'enfant interrogé. Les premiers nous ont beaucoup donné à réfléchir sur ce que l'on peut tirer de cette méthode, ses avantages, ses biais, ses limites, nous en avons d'ailleurs tiré tout un article réflexif paru dans la revue Children's Geographies. Les seconds ont fonctionné au-delà de ce que nous en espérions au départ, et très vite nous avons allongé leur durée. Mais là aussi de nombreuses questions se sont posées sur les photos (le choix du lieu, celui de la prise de vue, etc.) et sur le rapport des enfants à l'entretien, très variable d'un enfant à l'autre.

L'autre série d'enjeux, au-delà de la question de l'adaptation des dispositifs méthodologiques aux enfants, ce sont les enjeux éthiques. Les chercheurs des Children's Geographies se sont beaucoup interrogés sur leurs propres pratiques de recherche, conscients de mener leurs enquêtes auprès d'une population au statut spécifique, particulièrement vulnérable. Ils ont cherché à expliciter le plus clairement possible tout un ensemble de questions. Comment les relations de pouvoir entre enfants et adultes jouent-elles dans la relation d'enquête? Comment s'assurer du consentement éclairé des enquêtés à participer à la recherche ? Comment garantir leur anonymat et la confidentialité de leur contribution? Quel est le but de la recherche et quels intérêts sert-elle ? Comment s'assurer que la recherche et ses résultats ne nuiront pas aux enquêtés ? Ces questionnements ont fait l'objet de nombreuses publications à partir du début des années 2000, surtout dans la sphère scientifique anglophone, en lien avec la mise en place d'une régulation éthique des 
sciences sociales beaucoup plus précoce qu'en France, et qui vient aussi avec son lot de contraintes.

CR. Qu'est-ce que la géographie des enfants est, selon vous, en mesure de nous dire de la géographie des parents?

SLF. Vous posez ici la question très importante de l'articulation des géographies des enfants avec celles de leurs parents, et il faut bien comprendre qu'elle se pose dans les deux sens : comment les enfants structurent-ils ou, du moins, contribuent-ils à structurer le territoire de leurs parents, et comment inversement les parents contribuent-ils eux aussi à structurer le territoire de leurs enfants?

De nombreuses recherches, en sociologie particulièrement, ont mis en évidence l'impact des enfants sur les choix résidentiels des parents, tout particulièrement ceux des classes moyennes autour des enjeux scolaires. Notre recherche sur les quartiers gentrifiés a confirmé cela pour les territoires étudiés à Paris, Londres et San Francisco. Elle a montré aussi que les pratiques des parents se recentrent localement avec la naissance de leurs enfants, que l'espace et le temps de leur quotidien s'articule autour d'eux, et que leurs enfants sont l'occasion pour eux de nouvelles sociabilités locales, susceptibles de modifier leurs représentations et leurs pratiques du quartier et de la ville. Mais ce qui est intéressant, c'est d'observer les recoupements et les décalages entre les territoires des enfants et ceux des parents. Notre recherche a révélé par exemple qu'à l'échelle du quartier, les pratiques et les sociabilités des parents et des enfants peuvent être très imbriquées : les trajets pour l'école, les courses, le parc, etc. donnent lieu à des pratiques conjointes qui peuvent évoluer en véritables traditions familiales, des "sociabilités de famille" se développent, renforcées par la proximité socio-spatiale et par les amitiés croisées des enfants et des parents. On observe une plus ou moins grande continuité entre le quartier des enfants et celui des parents. Mais dans l'ensemble, les pratiques et sociabilités des enfants sont plus intenses que celles de leurs parents dans le quartier.

À l'inverse, les géographies des enfants sont très nettement structurées par les parents et leurs stratégies éducatives, comme le montrent les travaux de Gill Valentine ou les vôtres par exemple ! Elles dépendent des représentations que les parents se font du quartier, de l'importance qu'ils accordent à la vie de quartier de leurs enfants ou à leur intégration à une communauté locale. Dans les quartiers gentrifiés, notre recherche a montré que les territoires des enfants dépendent aussi du rapport des parents à la mixité sociale : non seulement ils n'en ont pas tous la même conception, mais ils lui confèrent un rôle différent dans l'éducation de leurs enfants - certains le considèrent comme simple décor, alors que d'autres lui donnent une dimension éducative forte. Globalement, cela se traduit par un fort encadrement des usages du quartier, par un contrôle de leurs pratiques, de leurs relations sociales et de leurs activités. Mais cet encadrement parental varie selon les lieux et les temps (du logement au quartier, à la ville ou au village, et jusqu'aux territoires des vacances). Et bien sûr, on observe des variations sensibles dans ces stratégies en fonction des milieux sociaux - comme l'a magistralement démontré la sociologue américaine Annette Lareau-, mais aussi des profils sociaux des quartiers de résidence, des contextes nationaux, du genre (celui des enfants, mais aussi celui des parents), de l'âge, de la place dans la fratrie, de la composition familiale, etc. Les entretiens menés avec les enfants font souvent explicitement référence aux discours et consignes parentales pour justifier le degré d'autonomie spatiale dont ils 
bénéficient par exemple. Mais ils permettent aussi de saisir les marges de manœuvre que les enfants se construisent parfois, en négociant avec leurs parents ou à leur insu, pour déployer des pratiques et des sociabilités qui leur sont propres, à l'école, sur le chemin de l'école, au parc, ou sur leur lieu de vacances.

Quoiqu'il en soit, je crois que ce qu'il faut retenir, c'est cette idée de co-construction et d'imbrication des géographies des enfants et de leurs parents, et aussi des variations qui nuancent toujours les grandes tendances observées, d'où l'importance de parler de " géographies », d'« enfants » et de " parents » au pluriel.

CR. Vous l'évoquiez tout à l'heure, vous avez participé à deux recherches collectives portant sur les expériences enfantines de la ville et du quartier. Pourriez-vous présenter ces recherches?

SLF. La première recherche était intitulée «Les enfants et la mixité sociale dans les quartiers gentrifiés à Paris, Londres et San Francisco ». Je l'ai menée avec JeanYves Authier et Frédéric Dufaux, dans une équipe interdiscipinaire associant deux géographes et un sociologue. Deux d'entre nous s'intéressaient particulièrement à la question de la gentrification, et c'est de cette perspective scientifique qu'a émergé cette recherche initialement. Comme Tim Butler l'avait bien rappelé, la gentrification est certes globale, mais elle produit non pas des quartiers gentrifiés homogènes et qui suivraient une évolution linéaire uniforme, mais au contraire des quartiers aux profils et aux «atmosphères » variés, que les gentrifieurs sélectionnent aussi en fonction de leurs attentes. Or, nous étions frappés par l'existence de quartiers gentrifiés familiaux, c'est-à-dire où les enfants étaient à la fois surreprésentés en termes de population, très visibles dans l'espace public, et très présents dans les représentations collectives des habitants, dans différentes villes que nous avions pu observer. Plusieurs travaux, comme ceux des sociologues Agnès Van Zanten ou Marco Oberti par exemple en France, s'étaient concentrés sur les stratégies résidentielles des parents en lien avec leurs stratégies éducatives, pour montrer leurs impacts sur la production de la gentrification ou de la ségrégation. Mais rien n'avait été fait encore sur les manières d'habiter et de cohabiter des jeunes enfants et de leurs familles, dans ces contextes de mixité sociale. Dans cette perspective, nous avons donc monté un dispositif de recherche en partant des écoles primaires des quartiers gentrifiés choisis à Paris, Londres et San Francisco. Nous avons sélectionné une école publique et une école primaire privée dans chaque quartier, de manière à pouvoir saisir certains enjeux locaux d'évitement scolaire autour de la question de la mixité sociale. Dans chaque école, comme je l'ai évoqué plus haut, nous avons conduit un « atelier quartier » auprès d'au moins une classe de la fin du cycle primaire, grâce auquel nous avons collecté à la fois des dessins du quartier vu par les enfants, des entretiens individuels auprès de ces enfants, ainsi que des entretiens auprès des enseignants et des parents. Cette recherche a mis en évidence la place centrale des enfants dans la vie de ces quartiers, elle a révélé la manière dont les pratiques et les représentations du quartier des parents et celles des enfants sont co-construites, imbriquées mais sans jamais être identiques, et, enfin, elle a mis au jour la grande diversité des rapports à la mixité des enfants et de leurs parents en fonction des caractéristiques sociales des familles, qui jouent des lieux (école, espace public, logement, etc.) et des temps pour déployer leur rapport à la mixité sociale.

À l'issue de cette recherche, nous avons souhaité poursuivre avec Jean-Yves Authier, Anaïs Collet et Isabelle Mallon ce questionnement sur les vies quotidiennes des 
enfants dans la ville et sur leurs manières d'habiter et de cohabiter dans deux autres types de quartiers socialement contrastés : des quartiers populaires d'un côté, et des quartiers bourgeois de l'autre. Nous avons restreint la comparaison à deux des trois villes initiales, Paris et San Francisco, et nous avons mis en place un dispositif d'enquête comparable à celui de la première recherche (un atelier quartier dans chaque école, pour collecter des dessins et des entretiens auprès d'enfants du même âge, ainsi que des entretiens avec les enseignants et les parents). Avec cette deuxième enquête, l'objectif est double. Il s'agit d'une part de saisir la différenciation spatiale des représentations, des pratiques et des sociabilités des enfants et de leurs familles, d'une ville à l'autre, et au sein d'une même ville, d'un quartier à l'autre - alors que d'autres recherches ont montré les différenciations des enfants selon leur milieu social ou leur genre. D'autre part, elle vise à interroger le rôle de l'espace (un quartier aux caractéristiques socio-spatiales bien définies) dans la socialisation des enfants, c'est-à-dire dans le processus d'apprentissage et d'intériorisation des valeurs, des normes et des rôles qui régissent le fonctionnement de la vie sociale. Cette recherche a donné lieu à de premiers résultats notamment sur les apprentissages différenciés de l'autonomie spatiale des enfants, sur leurs représentations de leur quartier, ou encore sur leurs relations de voisinage.

Dans ces deux enquêtes, quel a été l'apport du choix de la comparaison, entre différentes villes d'abord, mais aussi entre différents quartiers au sein d'une même métropole?

Pour la première enquête, la perspective comparative s'est immédiatement imposée dans la mesure où c'est l'observation directe de quartiers gentrifiés caractérisés par une forte visibilité de familles avec jeunes enfants, à Paris et à San Francisco, qui a fait émerger notre questionnement sur ce type de quartier presque totalement ignoré par la littérature sur la gentrification. En ajoutant Londres, nous obtenions un panel de villes inscrites au cœur de métropoles mondiales, cosmopolites, et situées dans trois pays occidentaux distincts, notoirement identifiées comme étant soumises à de puissants processus de gentrification (largement étudiés par la communauté scientifique locale et internationale), et toutes affectées par le déclin du nombre de familles avec jeunes enfants. La comparaison visait donc à mettre en avant les tendances communes concernant la place des enfants et de leurs familles dans le processus de "gentrification généralisée " pour reprendre les termes de Neil Smith. En même temps, il s'agissait d'être en mesure de nuancer ces tendances générales en montrant le rôle des contextes nationaux et locaux spécifiques dans les différenciations observées (chacun des terrains est le produit d'une histoire et d'une conception de la société, de la ville, de la famille, de l'école qui lui sont propres).

Avec la deuxième enquête, nous avons porté la démarche comparative à un autre niveau - tout en laissant de côté le cas de la métropole londonienne pour des raisons de faisabilité. Il s'agissait de comparer cette fois non plus seulement un type de quartier dans trois métropoles, mais deux types de quartiers (populaires et bourgeois) au sein de deux métropoles différentes (Paris et San Francisco), ce qui à notre connaissance est une méthode inédite dans les recherches portant sur ce domaine. La mise en œuvre de cette double comparaison comporte plusieurs avantages. D'abord, elle permet d'insister sur le quartier comme échelle d'analyse pertinente pour saisir les vies quotidiennes des enfants en ville: il s'agit de saisir dans quelle mesure et de quelle manière le quartier structure les manières d'habiter et de cohabiter des enfants en ville, à San Francisco ou à Paris, dans les quartiers 
populaires ou dans les quartiers bourgeois. Ensuite, cette double comparaison permet d'éviter à la fois la tentation d'un surdéterminisme culturel (à laquelle on est soumis lorsque l'on compare un même type de quartier dans différents contextes nationaux) et celle d'un surdéterminisme social (lorsqu'on compare trois types de quartiers dans un même contexte national). C'est ce que montre par exemple notre analyse de l'autonomie spatiale des enfants. D'une part, en mettant en évidence un certain nombre de tendances communes à tous les terrains (à savoir une autonomie spatiale globalement restreinte, nuancée par l'existence et la diversité des expériences d'autonomie, et la complexité du rapport des enfants à leur propre autonomie), la double comparaison donne finalement des fondements plus solides à une montée en généralité. D'autre part, elle permet de mettre en lumière des différenciations non seulement terme à terme (les enfants de San Francisco jouissent d'une autonomie spatiale moindre que ceux de Paris, quel que soit leur milieu social; et les enfants issus des milieux populaires ont des pratiques spatiales plus autonomes que ceux issus des catégories supérieures, quelle que soit la ville observée), mais aussi des différenciations emboîtées ou à géométrie variable en fonction des registres observés (les enfants du quartier bourgeois de San Francisco ont l'autonomie la plus restreinte; et en ajoutant des variables, ce sont les garçons du quartier populaire parisien, issus de familles monoparentales et dont le parent a un métier réduisant le temps de présence dans le foyer, qui jouissent de la plus grande autonomie). Finalement, comparer permet non seulement de mettre en évidence des régularités et des différenciations, mais aussi de penser autrement chaque réalité observée.

CR. En quoi les résultats de ces recherches nous disent-ils quelque chose des transformations de la ville et des grands processus urbains contemporains?

SLF. Comme je le disais plus tôt, c'est vraiment à partir du champ de la gentrification (un terme qui désigne, pour faire court, le processus de transformation socio-spatiale des quartiers centraux populaires par l'afflux de ménages des classes moyennes supérieures) que notre première recherche sur les enfants s'est progressivement construite, et non l'inverse. Nous n'avions pas d'expertise particulière, au départ, dans les Children's Geographies. C'est en tant que spécialistes de la gentrification (pour deux des trois personnes de l'équipe initiale) que nous avons compris l'importance d'étudier cette catégorie de population globalement négligée par les études urbaines et que nous sommes allés puiser dans les ressources (théoriques et méthodologiques) des Children's Geographies et plus largement des Children's Studies. D'abord, parce que les parents constituent des acteurs importants de la transformation des villes et de leurs quartiers (ceux qu'ils quittent et ceux dans lesquels ils s'installent). Quelques chercheurs avaient montré le poids de leurs stratégies éducatives dans leurs motivations résidentielles, et leur rôle, en bout de chaîne, dans la production de la gentrification ou de la ségrégation des espaces urbains. Notre recherche, à partir d'entretiens menés dans les quartiers gentrifiés, confirme que les enfants constituent un critère de choix majeur du logement et du quartier, y compris pour les parents les plus anciennement installés, même si ces motivations varient dans le temps, en fonction de la date d'arrivée dans le quartier (et donc de ses caractéristiques à ce moment de son évolution) : les écoles, les parcs et jardins et l'atmosphère familiale sont cités comme les principaux atouts. Les enfants constituent donc indirectement des facteurs de gentrification de certains quartiers. Autrement dit, nous sommes venus à la géographie des enfants d'abord par le biais de la géographie des parents! 
En même temps, nous étions intéressés par les quartiers tels qu'ils sont produits par la gentrification: quel impact les transformations des villes (la gentrification en l'occurrence) ont-elles sur le rapport des habitants à leur quartier et à leur ville, sur leurs manières d'habiter et de cohabiter? Et, alors qu'on commence à saisir le rôle des enfants (et de leurs parents) dans ce processus, comment vivent-ils au quotidien la gentrification et la mixité sociale qui la caractérise, comment se la représententils, quelles pratiques et quelles sociabilités déploient-ils? La deuxième recherche est portée par les mêmes questionnements sur les transformations urbaines: comprendre d'une part la place des enfants (et de leurs parents) dans la production non plus de la gentrification mais de la ségrégation (sociale) - aux deux extrémités du spectre social: dans les quartiers populaires et dans les quartiers bourgeois - et, d'autre part, saisir les manières dont ces quartiers socialement ségrégués sont perçus, interprétés, travaillés, éventuellement détournés, par les enfants (et leur parents) dans leurs pratiques quotidiennes. La géographe néerlandaise Lia Karsten est l'une des rares autres chercheuses à ce jour à avoir interrogé la notion de ségrégation à partir de la question du rapport des enfants au quartier et à la ville dans deux articles portant sur Amsterdam : elle montre notamment que plus que l'école, qui est incontestablement ségréguée, c'est surtout le déclin de l'autonomie spatiale des enfants dans la ville qui explique l'homogénéisation croissante des réseaux de sociabilité des jeunes citadins d'aujourd'hui.

\section{BIBLIOGRAPHIE}

Authier J.-Y., Collet A., Lehman-Frisch S., Mallon I., 2019. Comparer les vies urbaines des enfants à Paris et à San Francisco. In Authier J.-Y., Baggioni V., Cousin B., Fijalkow Y., Launay L., La comparaison internationale en sociologie urbaine. Paris, La Découverte, p. 209-226.

Authier J.-Y., Lehman-Frisch S., 2012. Il était une fois... des enfants dans des quartiers gentrifiés à Paris et à San Francisco. Actes de la Recherche en Sciences Sociales, ${ }^{\circ}{ }^{195}$, p. 59-73.

Authier J.-Y., Lehman-Frisch S., 2013. Le goût des autres : Gentrification Told by Children. Urban Studies, vol. 50, nº 3, p. 994-1010.

Butler T., Robson G., 2003. London Calling : The Middle Class and the Re-Making of Inner London. Oxford, New-York, Berg.

Karsten L., 1998. Growing up in Amsterdam : Differentiation and Segregation in Children's Daily Lives. Urban Studies, vol. 35, $n^{\circ}$ 3, p. 565-581.

Karsten L., 2010. Children's Social Capital in the Segregated Context of Amsterdam : An Historical-Geographical Approach. Urban Studies, vol. 48, nº 8, p. 1-16.

Lareau A., 2003. Unequal Childhoods : Class, Race and Family Life. Los Angeles, University of California Press. 
Lehman-Frisch S., Vivet J., 2011. Géographies des enfants et des jeunes. Carnets de Géographes [En ligne], $\mathrm{n}^{\circ}$ 3. URL: http://journals.openedition.org/cdg/2074 - DOI: https://doi.org/10.4000/cdg. 2074

Lehman-Frisch S. (dir.), Authier J.-Y., Dufaux F., 2012. Les enfants et la mixité sociale dans les quartiers gentrifiés à Paris, Londres et San Francisco. Caisse Nationale des Allocations Familiales, Dossiers d'études, $\mathrm{n}^{\circ} 153$.

Lehman-Frisch S., Authier J.-Y., Dufaux F., 2012. « Draw me your Neighborhood » : A Gentrified Paris Neighbourhood Through its Children's Eyes. Children's Studies, vol. 10, n 1, p. 17-34.

Oberti M., 2007. L'école dans la ville : ségrégation-mixité-carte scolaire. Paris, Presses de Sciences Po.

Rivière C., 2017. Du domicile à la ville : étapes et espaces de l'encadrement parental des pratiques urbaines des enfants. Espaces et sociétés, n 168-169, p. 171-188.

Smith N., 2003. La gentrification généralisée : d'une anomalie locale à la régénération urbaine comme stratégie urbaine globale. In Bidou-Zachariasen C. (dir.), Retours en ville. Des processus de " gentrification » urbaine aux politiques de « revitalisation » des centres. Paris, Descartes et Cie, p. 45-72.

Valentine G., 2004. Public Space and the Culture of Childhood. London, Ashgate.

Van Zanten A., 2009. Choisir son école : les stratégies éducatives des classes moyennes, Paris, PUF.

\section{AUTEURS}

\section{SONIA LEHMAN-FRISCH}

Sonia Lehman-Frisch est Professeur à l'Université Paris Nanterre. Elle a notamment publié : - Authier J.-Y., V. Bathellier, Lehman-Frisch S. (coord.), 2016. La place des enfants et des adolescents dans les espaces urbains. Les Annales de la recherche urbaine, $\mathrm{n}^{\circ} 111$.

- Authier J.-Y., Lehman-Frisch S., 2014. Exposer ses enfants à la mixité sociale : discours et pratiques des parents de classes moyennes supérieures dans deux quartiers gentrifiés de Paris et San Francisco. Politiques Sociales et Familiales [En ligne], n 117, p. 59-70. URL: https://www.caf.fr/ sites/default/files/cnaf/Documents/Dser/PSF/117/PSF117_1_JYAuthier_SLehmanFrisch.pdf - Authier J.-Y., Lehman-Frisch S., 2012. Variations sur un thème : Les manières d'habiter des enfants dans les quartiers gentrifiés à Paris, Londres et San Francisco. Métropoles [En ligne], $\mathrm{n}^{\circ}$ 11. URL: http://journals.openedition.org/metropoles/4584 - DOI: https://doi.org/10.4000/ metropoles. 4584

\section{CLÉMENT RIVIÈRE}

Clément Rivière est maître de conférences en sociologie à l'Université de Lille et chercheur au Centre de Recherche "Individus, Epreuves, Sociétés". 